bodied speckled snakes, known as king-snakes, found to be fangless, and consequently without venom, were duly installed as members of the family. Some uneasiness was perceivable among the older members, but no attempt was made to destroy the intruders, though they might have been killed instanter. The next morning four of the venomous serpents were found to have been destroyed by the king-snakes, and one was still within their coil, and the two remaining ones would make no effort at self-defence. A large rattlesnake seemed stupid and indifferent to his fate. He could not be made to threaten or give warning even with his rattles. The smallest king-snake was afterwards inoculated with the poison of one of the serpents be had destroyed, and died immediately after-thus evincing that they must have exercised some power besides physical force to overcome their fellow creatures.

In short, the results of a great number of experiments performed with the venom of a great variety of serpents, seem to lead to the following conclusions :-

Ist. That the venom of all serpents acts as a poison in a similar manner.

2d. That the venom of some varieties is far more active than that of others.

3d. That a variety of the colluber, known as the cotton-mouth, is the most venomous serpent in Arkansas.

4th. That the venom of serpents destroys all forms of organized life, vegetable as well as animal.

5 th. That alcohol, if brought in contact with the venom, is, to a certain extent, an antidote.

6th. That serpents do possess the power of fascinating small animals, and that this power is identical with mesmerism.

$7 \mathrm{th}$. That the blood of sinall animals, destroyed by the venom of serpents, bears a close resemblance to that of animals destroyed by lightning or hydrocyanic acid; it loses its power of coagulation and cannot be long kept from putrefaction._St. Louis Med. and Surg. Jour.

\title{
A SINGULAR CASE-HYDROPHOBIC SYMPTOMS.
}

BY J. E. THOMPSON, M.D., OF MIssoURI.

[Conmunicated for the Boston Medical and Surgical Journa].]

March 30, 1854, I was called professionally to see John Connel, ætas 35, an Irish laborer, of good habits and constitution, rather corpulent, and of great muscular strength. Found the patient under much nervous excitement, naked, and walking his room to and fro. Notwithstanding all the windows were raised, and the doors thrown open, he would every few minutes exclaim, "Great God! I am smothering! I am smothering" ! His eyes were red as though under the influence of intoxication ; countenance greatly dejected; pulse 128, small and thready: respiration difficult; bowels distended; skin hot and dry, with a thin, lightcolored coating upon the tongue. No local injury could be found. 1 immediately threw several pailfuls of ice-water upon his body, which, after a 
moment, allayed his excitement, upon which he exclaimed, " $O$ that $I$ could lie in a river of ice-water!" He complained of great thirst, entreating his friends "for God's sake to give him just one drop of cold water to cool his parched tongue" ! which they refused. I allowed him a cup; but no sooner bad he raised it to his lips, than a violent spasm seized him in the muscles of the throat. I gave of sp. æth. sulph. comp., 3 jss., to be repeated at every recurrence of the paroxysm. The fit lasted some five minutes. He sprang from the bed, which we had placed him upon while in the fit, as though frightened, saying he was smothering; upon no consideration could he be induced to remain. I ordered the following :-R. Sulph. morph., gr. j. ; Pulv. ipecac. et opii, grs. x. Misce. Fiat pulvis, in chartulas sex dividendus. One to be taken in syrup every third bour. Cold applications to be applied to the head and neck every few hours.

31 st.-Has not slept; walked to and fio all night ; spasms recuned three times, when attempting to drink water; thirst intense; complete aversion to food of all kinds; has not ate anything since the 29 th ; countenance anxious; respiration difficult ; pulse 124, small ; considerable irritability of the nervous system; bowels distended; pain in the back and head. Gave of R. Hyd. chlo. mit., grs. viij.; pulv. rhei, gr's. v.; pulv, aloes, grs. ij. Misce. Quieting pulv. discontinued, and pil. hyoscy. no. ij. given every hour till sleep is produced. Cold lotion continued.

April 1st.-Has not slept. Has had four alvine discharges; has neither ate nor drank anything; pulse 115. Other symptoms pretty much as yesterday. Pil. hyoscy. continued.

2d.-Has had three alvine discharges; pulse 100 ; can lie in bed without much inconvenience, but cannot sleep; has taken no food for three days ; thirst intense, but spasm!s recur when any attempts are made to take water; countenance dejected. Treatment continued.

3d.-Has slept about ten minutes, the first minute's sleep since March 29 th ; paroxysms recurred twice, but slight, when attempting to drink ; does not experience much difficulty in breathing. Drank a little beef-tea. Has had two alvine discharges; pulse 90 , full; tongue covered in the centre with a thick, dark-yellow coating, red and sharp at the tip. Gave of the following, R. Sulph. quin., gr. xv. ; ferri ferrocy., grs. xxx.; pulv. acacia, q. s. Misce, et fiat massa in pilulas duodecim. dividenda. 'Three to be taken every four hours. Pil. hyoscy. no. j. to be taken every hour.

4th.-Slept about two hours through the night ; cannot drink water yet ; took some beef-tea and rice; pulse 80 ; respiration natural: had two alvine discharges. Treatment continued.

5th.-Slept well all night; took a cup of water, but was afraid to drink much. Improving rapidly.

6th.-Can drink without any difficulty. Patient said he was well, and he was discharged cured.

Remarks.- Since the occurrence of this case, I bave searched in journals and works on medical practice, and I cannot find a single case similar to the above. Some of my neighboring practitioners, to whom I 
have related it, say it " is emphatically hydrophobia." Others say not. But I must confess myself in a quandary. Is it hydrophobia? I could find no marks of wounds or local injuries likely to catse it. The patient says he "never saw a mad dog, let alone being bitten by one." Does idiopathic hydrophobia ever happen in the human subject? Would we not be justified, according to the diagnosis, in calling it typho-hydrophobic congestion?

Osage Co., Mo., June 6th, 1854.

\section{THE VENTILATION OF HOUSE-DRAINS.}

BY FRANCIS MINOT, M.D., BOSTON.

[Communicated for the Boston Medica] and Surgica] Journal.]

THE occurrence of several cases of cholera in Boston, within the last few days, has called special attention to the sanitary condition of the city, and the most energetic and complete system of purifying the streets, cellars and drains will undoubtedly be put into operation by the city authorities. Should we be again visited by an epidemic, as in the year 1849 , we have every reason to hope that, so far as public sanitary measures can avail, the disease will fall with comparative lightness upon us, and that Boston will maintain the high reputation she has always enjoyed for being a clean and healthy city.

There is an evil, however, which exists to some extent among us, and which may, under favorable circumstances, as will be amply shown, become a fruitful source of disease. We refer to the absence of ventilation in house-drains and water-closets. The house-drains of this city are either directly connected with the street sewers, or communicate with them by means of a cess-pool, which is intended to prevent the reflux of air towards the house, while it permits the flow of the liquid part of the sulliage in the opposite direction. When the communication with the sewver is direct, the house is liable to be flooded with gas of the most offensive and deleterious properties, from various causes, of which a frequent one with us is a strong wind blowing into the sewer, when its mouth is exposed at low tide. A well-constructed cess-pool is an efficient remedy against this calamity, especially in a city like Boston, where the inclination of the soil is favorable to drainage, and where the sewers are well built, and well cleansed by the vast quantities of waste water allowed to run through thern.

Most of the better class of houses communicate directly with the main sewers by means of water-closets, which discharge their contents immediately into them, without the intervention of cess-pools, the only protection against the reflux of foul air into the house being the trap of the water-closet, which is an insufficient barrier against a strong pressure from without. This is evident from the fact that when the machine is not in action, a gurgling sound is occasionally heard in the soil-pan, the water suddenly sinks below its former level, and sometimes even wholly disappears, showing that the external pressure has been able to force the gas through the water. This evil, however, is only occasional, being 